\title{
Probing Structural Attributes for Li-Argyrodite as a Fast lon Conductor using Neutron Powder Diffraction \\ Po-Hsiu Chien ${ }^{1}$, Xuyong Feng ${ }^{2}$, Jue Liu ${ }^{3}$ \\ ${ }^{1}$ No affiliation given ${ }^{2}$ The Walker Department of Mechanical Engineering, The University of Texas at Austin, ${ }^{3}$ Neutron Scattering Division, Oak Ridge National Laboratory chiena@ornl.gov
}

Driven by technological importance, the development of all-solid-state lithium-ion batteries has awakened the pursuit of solid electrolytes with superior $\mathrm{Li}+$ conductivity. Li-Argyrodite is a unique player in the field of solid electrolytes because this sulfide-based material was shown to achieve an ionic conductivity of $24 \mathrm{mS} / \mathrm{cm}$ at room temperature. This record, which even outperforms the liquid electrolyte $(\sim 10 \mathrm{mS} / \mathrm{cm}$ at r.t.), calls for an understanding of the origin of fast ion conduction in Li-Argyrodite. In this work, we employ variable-temperature neutron diffraction and impedance spectroscopy to investigate the structural attributes for $\mathrm{Li}+$ transport properties in Li-Argyrodite, Li6PS5X (X = Cl, Br, and I). Structural analyses based on the Rietveld refinements, maximum entropy method (MEM) analysis, and bond valence site energy (BVSE) reveal that $\mathrm{Li}+$ has the potential to take an interstitial site $16 \mathrm{e}$, enabling a cage-to-cage three-dimensional $\mathrm{Li}+$ conduction via $48 \mathrm{~h}-16 \mathrm{e}-48 \mathrm{~h}$ pathway. In addition, we find that the anion site disorder between $\mathrm{S} 2-$ and $\mathrm{X}_{-}$, which is commonly perceived as the key factor to promote $\mathrm{Li}+$ conductivity, is fundamentally correlated with the negative charge distribution over the anion $4 \mathrm{a}$ and $4 \mathrm{c}$ sites. Learned from this understanding, we further show that tuning the negative charge ratio of $4 \mathrm{a}$ and $4 \mathrm{c}$ to unity is an effective approach to realizing higher $\mathrm{Li}+$ conductivity in $\mathrm{Li}$.7 $7 \mathrm{PS} 4.7 \mathrm{ClBr} 0.3$. 